\title{
Final state interactions effects on kinetic energy sum spectra in nonmesonic weak decay
}

\author{
C. Barbero \\ Departamento de Física, Universidad Nacional de La Plata, C. C. 67, 1900 La Plata, Argentina
}

A. Mariano

Departamento de Física, Universidad Nacional de La Plata, C. C. 67, 1900 La Plata, Argentina, Instituto de Física La Plata, CONICET, 1900 La Plata, Argentina

S.B. Duarte

Centro Brasileiro de Pesquisas Físicas, Rua Dr Xavier Sigaud 150, CEP 22290-180, Rio de Janeiro-RJ, Brazil

(Received on 31 December, 2009)

\begin{abstract}
We analyze the effect of final state interactions (FSI) on coincidence spectra in nonmesonic hypernuclear weak decay, $\Lambda N \rightarrow n N$, as a function of the kinetic energy sum, $E_{n N}=E_{n}+E_{N}$, both for $n p$ and $n n$ events. Adopting a formalism recently developed, the effects of FSI originated from the interaction between the outgoing nucleons and those in the residual core are included analytically in a very simple way within the eikonal approximation through the modification of the emerging particles momenta. Numerical results are shown for ${ }_{\Lambda}^{5} \mathrm{He}$ and ${ }_{\Lambda}^{12} \mathrm{C}$ hypernucleus. We found that coincidence spectra are only slightly modified and the disagreement between theory and experimental data still persists, mainly for $n n$ events in ${ }_{\Lambda}^{5} \mathrm{He}$ where enough statistics exists. We conclude that admixtures of excitations in the final state produced by FSI need to be added to our approach in order to improve the agreement with data.
\end{abstract}

Keywords: Hypernuclei, Sum spectra, Final state interactions.

\section{INTRODUCTION}

The nonmesonic hypernuclear weak decay (NMHD) $\Lambda N \rightarrow N N$ is the dominant channel for a $\Lambda$ particle decaying inside nuclear medium because the free decay through the mesonic mode $\Lambda \rightarrow \pi N$ is Pauli blocked. That NMHD mode offers a good opportunity to scrutinize the strangenesschanging $|\Delta S|=1$ nonleptonic weak interaction between hadrons. As such, it is useful to advance in the knowledge of the involved physics. Along the last four decades there has been a continuous interest in this decay mode strongly motivated by the disagreement between theoretical results and experimental data for the different observables: i) the total nonmesonic decay width, $\Gamma_{N M}=\Gamma_{n}+\Gamma_{p}$, being $\Gamma_{n}$ and $\Gamma_{p}$ the rates for the neutron and proton induced decay channels, $\Lambda n \rightarrow n n$ and $\Lambda p \rightarrow n p$, respectively; ii) the neutron to proton ratio, $\Gamma_{n / p} \equiv \Gamma_{n} / \Gamma_{p}$; iii) the intrinsic asymmetry parameter, $a_{\Lambda}$; iv) the kinetic energy spectra for protons and neutrons, $S_{N}\left(E_{N}\right)$; and v) the double coincidence nucleon spectra, $S_{n N}\left(\cos \theta_{n N}\right)$ and $S_{n N}\left(E_{n}+E_{N}\right)$, showing the angular and kinetic energy sum distributions of the emitted nucleons.

Several important advances have been made in recent years regarding the determination of $\Gamma_{n / p}$, both from the theoretical and experimental side. In fact, the recent measurements of single and double coincidence nucleon spectra in different experiments have been somehow managed to derive values of that observable, which indicates the necessity of having a good knowledge about the spectra in NMHD. Between those experiments we mention: i) the new high quality measurements of single-nucleon spectra done in Refs. [1-5], and ii) the double-coincidence nucleon spectra from Refs. $[4,6-10]$. It is also important to mention the new experiments planned at J-PARC where the statistics will be increased much higher and the spectra will be observed clearly [11]. Additionally, on the theoretical hand some evaluations have been performed in order to analyze the spectra [12-18]. Related with the coincidence $n N$ spectra, in which we will concentrate from here on, it has been repeatedly mentioned in the literature that: i) the bump observed in the experimental $S_{n n}\left(E_{n}+E_{n}\right)$ spectrum at low energies is not reproduced by the theory, which may be indicative of $n n$ coincidences originated from sources other than $\Lambda n$ decays; and ii) differences between theoretical calculations and experimental data may be the result of final state interactions (FSI) in the $n p$ and $n n$ channels and/or two-nucleon-induced decay mode $(\Lambda N N \rightarrow N N N)$.

Particularly, we will pay special attention to the work from Ref. [16] where is developed a model for the evaluation of coincidence spectra which includes, in the framework of the independent particle shell model, the effects of the recoil of the residual nucleus and of the spreading in strength of the deep-hole states on NMHD observables. There is concluded that: i) that model is the appropriate lowest-order approximation for the theoretical calculations of the kinetic energy sum spectra in NMHD, and ii) it is in comparison to that picture that one should to appraise the effects of FSI and of twonucleon-induced decay mode. On the other hand, very recently we have presented a simple and analytical formalism to evaluate FSI experimented by the two nucleons leaving the residual nucleus after the primary nomesonic hyperonnucleon decay process [19]. There, an approach based on the eikonal approximation is developed and an optical potential is introduced to represent nuclear medium and to describe the nucleon-nucleus dispersion process along the nucleon outgoing path. In that work we analyze the effect of FSI on integrated observables such as the number of emitted pairs of nucleons and the asymmetry parameter, but not over the spectra. Subsequently, a similar idea has been applied and a relativistic model for NMHD which includes the effects of FSI through an energy-dependent complex optical potential has been used to study integrated observables and the kinetic energy and angular spectra [18].

All the above mentioned arguments have motivated us to analyze the effect of FSI on the kinetic energy sum spectra 
adopting the eikonal approach. In Sect. 2. we will briefly describe the procedure introduced in Ref. [19] to incorporate FSI to the formalism developed in Ref. [16]. Numerical results and summarizing conclusions are drawn in Sect. 3.

\section{FORMALISM}

The decay rate for the primary NMHD of an initial hypernucleus ${ }_{\Lambda}^{A+1} \mathrm{X}$ (with spin $J_{I} M_{I}$ and energy $E_{J_{I}}$ ) to a residual nucleus ${ }_{\Lambda}^{A} \mathrm{Y}$ (with several states having spin $J_{F} M_{F}$ and energies $E_{v_{F} J_{F}}$ ) plus two free nucleons (with spins, isospins and momenta $s_{N}, t_{N}$ and $\mathbf{p}_{N}$, respectively, for $N=n, p$ ) is evaluated from the expression [20-22]

$$
\begin{aligned}
& \Gamma_{\bar{N}} 2 \pi \sum_{s_{n} s_{N} t_{n} t_{N} v_{F} J_{F} M_{F}} \int\left|\left(\mathbf{p}_{n} s_{n} t_{n} \mathbf{p}_{N} s_{N} t_{N} ; v_{F} J_{F} M_{F}|V| J_{I} M_{I}\right)\right|^{2} \\
& \quad \times \delta\left(E_{n N}+E_{r}-\Delta_{V_{F} J_{F}}\right) \frac{d \mathbf{p}_{n}}{(2 \pi)^{3}} \frac{d \mathbf{p}_{N}}{(2 \pi)^{3}}
\end{aligned}
$$

Here $E_{n N}=\frac{p_{n}^{2}}{2 m_{N}}+\frac{p_{N}^{2}}{2 m_{N}}$ is the total kinetic energy of the outgoing primary pair with $m_{N}$ being the nucleon mass, $E_{r}=\frac{\left|\mathbf{p}_{n}+\mathbf{p}_{N}\right|^{2}}{2 m_{N}(A-1)}$ is the recoil energy, $\Delta_{V_{F} J_{F}}=E_{J_{I}}-E_{\mathrm{V}_{F} J_{F}}+\Delta$ with $\Delta=m_{\Lambda}-m_{N}$ is the released energy being $m_{\Lambda}$ the $\Lambda$ mass, and $V$ is the transition potential used to describe the primary nonmesonic decay process.

$$
<\mathbf{r}_{n}\left|\mathbf{p}_{n}><\mathbf{r}_{N}\right| \mathbf{p}_{N}>=e^{i \alpha_{N} \mathbf{p} \cdot \mathbf{r}} e^{i \alpha_{N} \mathbf{P} \cdot \mathbf{R}}
$$

where we have made the change to relative and center of mass variables $\left(\mathbf{p}=\frac{1}{2}\left(\mathbf{p}_{n}-\mathbf{p}_{N}\right), \mathbf{P}=\mathbf{p}_{n}+\mathbf{p}_{N}, \mathbf{r}=\mathbf{r}_{n}-\mathbf{r}_{N}\right.$ and $\left.\mathbf{R}=\frac{1}{2}\left(\mathbf{r}_{n}+\mathbf{r}_{N}\right)\right)$ and we have defined $\langle p\rangle^{2}=\left(\frac{p_{F}+p_{\max }}{2}\right)^{2}$, with $p_{F}=\sqrt{2 m_{N} \varepsilon_{F}}$ and $p_{\max }=\sqrt{2 m_{N} \Delta\left(\frac{A-1}{A}\right)}$ being the Fermi and maximum momentum allowed kinematically to nucleons, respectively. The effective strength dependence on the residual nucleus and nucleon isospins is contained in the potential $\tilde{V}_{N}$ which holds

$$
\tilde{V}_{N}=\left\{\begin{array}{lr}
\tilde{V}_{0} & \text { for } N=n \\
\tilde{V}_{0}+\frac{\tilde{V}_{1}}{2 A} & \text { for } N=p
\end{array},\right.
$$

for ${ }_{\Lambda}^{5} \mathrm{He}$ and

$$
\tilde{V}_{N}= \begin{cases}\tilde{V}_{0}-\frac{\tilde{V}_{1}}{2 A} & \text { for } N=n \\ \tilde{V}_{0}+\frac{V_{1}}{2 A} & \text { for } N=p\end{cases}
$$

For the determination of the matrix element $\left(\mathbf{p}_{n} s_{n} t_{n} \mathbf{p}_{N} s_{N} t_{N} ; v_{F} J_{F} M_{F}|V| J_{I} M_{I}\right)$ we follow the treatment developed in Ref. [19]. The effects of FSI are incorporated by using the final nucleon states constructed in the framework of the nonrelativistic eikonal approximation [23-27]. In coordinate representation the accumulated effect of the interactions felt by the emerging nucleons along their outgoing path inside the residual nucleus is included through the action of the operator factor $S^{\dagger}(\mathbf{r})$ on the unperturbed plane wave functions [19, 28]: $<\mathbf{r}_{n}\left|\mathbf{p}_{n}><\mathbf{r}_{N}\right| \mathbf{p}_{N}>=S^{\dagger}\left(\mathbf{r}_{n}, \mathbf{p}_{n}\right) S^{\dagger}\left(\mathbf{r}_{N}, \mathbf{p}_{N}\right) e^{i \mathbf{p}_{n} \cdot \mathbf{r}_{n}} e^{i \mathbf{p}_{N} \cdot \mathbf{r}_{N}}$, where $S(\mathbf{r}, \mathbf{p})=e^{-i \frac{m_{N}}{p} \int_{z p}^{+\infty} d z V_{\text {opt }}(\mathbf{b}, z)}$. Here $\mathbf{p}$ has been chosen along the $z$ axis, $\mathbf{r}=\left(\mathbf{b}, z_{p}=\hat{\mathbf{p}} \cdot \mathbf{r}\right)$ represents the nucleon location after the primary nomesonic decay and $(\mathbf{b}, z)$ the posterior collision point, being $\mathbf{b}$ the impact parameter. For the optical potential associated to the nuclear medium representation we adopt the simplified form $V_{\text {opt }}(\mathbf{r})=-\left(V_{0}+\frac{V_{1}}{A} \tau \cdot \mathbf{T}\right)$ inside the nucleus $(|\mathbf{r}| \leq R$, being $R=1.25 A^{1 / 3}$ fm the nuclear radius) with $V_{0}$ and $V_{1}$ representing the real isoscalar and isovector "depths", respectively, and $\tau$ and $\mathbf{T}$ the nucleon and residual nucleus isospin operators. After some simple and straightforward algebra, the emerging two nucleon wave functions can be written as [19]

$\alpha_{N}=\left(1+\frac{m_{N}}{<p>^{2}} \tilde{V}_{N}\right), \quad$ for $N=n, p$,

for ${ }_{\Lambda}^{12} \mathrm{C}$. Here $\tilde{V}_{0}$ and $\tilde{V}_{1}$ represent average constant values.

Next, we follow two steps [16]: i) we write Eq. (1) in terms of relative and center of mass variables, $\mathbf{p}$ and $\mathbf{P}$, respectively, and defining $\varepsilon_{p}=\frac{p^{2}}{m_{N}}, \varepsilon_{P}=\frac{P^{2}}{4 m_{N}} \frac{A+1}{A-1}$ and $E_{r}=\frac{P^{2}}{2 m_{N}(A-1)}$ we carry out the $\delta\left(\varepsilon_{p}+\varepsilon_{P}-\Delta_{V_{F} J_{F}}\right)$ integration; ii) we express the decay rate as an integral in the variable $E \equiv E_{n N}=\Delta_{\mathrm{V}_{F} J_{F}}-\frac{2}{A+1} \varepsilon_{P}$, and obtain the transition probability density $S_{n N}(E)$ by performing the derivative on $E, S_{n N}(E)=\frac{d \Gamma_{N}}{d E}$. Thus, the spectrum as a function of the kinetic energy sum is given by

$$
\begin{aligned}
S_{n N}(E) & =\frac{4 m_{N}^{3}}{\pi} \sqrt{(A+1)(A-1)^{3}} \sum_{v_{F} J_{F}} \sum_{S l L \lambda J T} \sqrt{\left(\Delta_{v_{F} J_{F}}-E\right)\left(E-\Delta_{v_{F} J_{F}}^{\prime}\right)} \\
& \times\left|\left\langle\left(\alpha_{N} p\right) l\left(\alpha_{N} P\right) L \lambda S J T \nu_{F} J_{F} ; J_{I}|V| J_{I}\right\rangle\right|^{2},
\end{aligned}
$$


with $\quad p=\sqrt{\frac{m_{N}(A+1)}{2}\left(E-\Delta_{v_{F} J_{F}}^{\prime}\right)}, \quad P=$ $\sqrt{2 m_{N}(A-1)\left(\Delta_{v_{F} J_{F}}-E\right)}, \quad \Delta_{v_{F} J_{F}}^{\prime}=\Delta_{V_{F} J_{F}} \frac{A-1}{A+1} \quad$ and the condition $\Delta_{v_{F} J_{F}}^{\prime} \leq E \leq \Delta_{v_{F} J_{F}}$. The nuclear moment is given by

$$
\begin{aligned}
& \left\langle\left(\alpha_{N} p\right) l\left(\alpha_{N} P\right) L \lambda S J T v_{F} J_{F} ; J_{I}|V| J_{I}\right\rangle=\hat{J}_{I}^{-1} \sum_{\mathrm{j}_{N}} f_{J}\left(\mathrm{j}_{N} v_{F} J_{F}\right) \\
& \times \mathcal{M}\left(\left(\alpha_{N} p\right) l\left(\alpha_{N} P\right) L \lambda S J T ; \mathrm{j}_{\Lambda} \mathrm{j}_{N} m_{t_{N}}\right)
\end{aligned}
$$

with $\mathrm{j}_{N} \equiv n_{N} l_{N} j_{N} t_{N}$ and $\mathrm{j}_{\Lambda} \equiv n_{\Lambda} l_{\Lambda} j_{\Lambda} t_{\Lambda}$ being the singleparticle states for the nucleon and lambda, respectively (we assume that the $\Lambda$ particle behaves as a $\left|\frac{1}{2},-\frac{1}{2}\right\rangle$ isospin particle in the $1 s_{1 / 2}$ level). We have defined

$$
f_{J}\left(\mathrm{j}_{N} \vee_{F} J_{F}\right)=(-)^{2 J_{F}} \hat{J}_{I}\left\{\begin{array}{ccc}
J_{C} & J_{I} & j_{\Lambda} \\
J & j_{N} & J_{F}
\end{array}\right\}\left\langle J_{C} \| a_{\mathrm{j}_{N} m_{t_{N}}}^{\dagger}|| v_{F} J_{F}\right\rangle,
$$

with $J_{C}$ being the core spin such that $\left|\left(J_{C} j_{\Lambda}\right) J_{I}\right\rangle$ and $[20,21]$

$$
\begin{aligned}
& \mathcal{M}\left(\left(\alpha_{N} p\right) l\left(\alpha_{N} P\right) L \lambda S J T ; \mathrm{j}_{\Lambda} \mathrm{j}_{N} m_{t_{N}}\right)=\frac{1}{\sqrt{2}}\left[1-(-)^{l+S+T}\right] \\
& \times\left(\left(\alpha_{N} p\right) l\left(\alpha_{N} P\right) L \lambda S J T|V| \mathrm{j}_{\Lambda} \mathrm{j}_{N} m_{t_{N}} J\right),
\end{aligned}
$$

corresponds to the two body matrix element between the bounded $\Lambda$-nucleon system and the two final unbounded nucleons.

In order to account for the nuclear structure of the residual nucleus we follow the procedure from [16], i.e., we consider the highly excited hole states to be quasistationary and described by Breit-Wigner distributions, whose widths are estimated from the experimental data. Concretely, the perturbed eigenkets $\mid v_{F} J_{F}>$ are constructed starting from the unperturbed basis $\mid i_{N} J_{F}>_{0}$ with $i_{N}=0,1, \cdots, n_{N}, n_{N}+1, \cdots$, where for $i_{N} \leq n_{N}$ we have simple doorway states $\left|j_{N} J_{F}\right\rangle$ whereas for $i_{N} \geq n_{N}+1$ we have more complicated bound configurations as well as those including unbound singleparticle states in the continuum. Thus, writing

$$
\left|v_{F} J_{F}>=\sum_{j_{N}} C_{j_{N}}^{v_{F} J_{F}}\right| j_{N} J_{F}>+\sum_{i_{N}=n_{N}+1}^{\infty} C_{i_{N}}^{\nu_{F} J_{F}} \mid i_{N} J_{F}>_{0},
$$

it is easy to see that only the ket $\mid j_{N} J_{F}>$ in this expansion will contribute to the matrix element $\left\langle J_{C} \| a_{\mathrm{j}_{N} m_{t_{N}}}^{\dagger}|| v_{F} J_{F}\right\rangle$ in Eq. (7). Additionally, because of the high density of states, the amplitudes $C_{j_{N}}^{v_{F} J_{F}}$ will be estimated phenomenologically converting the discrete energies $\Delta_{V_{F} J_{F}}$ into the continuous variable $\varepsilon$ and the discrete sum on $v_{F}$ into an integral on $\varepsilon$. This allows to write [16]

$$
\begin{aligned}
S_{n N}(E) & =\frac{4 m_{N}^{3}}{\pi} \sqrt{(A+1)(A-1)^{3}} \sum_{J_{F}} \sum_{S l L \lambda J T} \int_{-\infty}^{\infty} P_{j_{N} J_{F}}(\varepsilon) \sqrt{(\varepsilon-E)\left(E-\varepsilon^{\prime}\right)} \\
& \times\left|\left\langle\left(\alpha_{N} p\right) l\left(\alpha_{N} P\right) L \lambda S J T J_{F} ; J_{I}|V| J_{I}\right\rangle\right|^{2} d \varepsilon,
\end{aligned}
$$

where $p=\sqrt{\frac{m_{N}(A+1)}{2}\left(E-\varepsilon^{\prime}\right)}, P=\sqrt{2 m_{N}(A-1)(\varepsilon-E)}$, $\varepsilon^{\prime}=\varepsilon \frac{A-1}{A+1}$ and the condition $\varepsilon^{\prime} \leq E \leq \varepsilon$ has to be fulfilled. Here $P_{j_{N} J_{F}}(\varepsilon)=\left|C_{j_{N} J_{F}}(\varepsilon)\right|^{2} \rho_{J_{F}}(\varepsilon)$ is the strength function being $\rho_{J_{F}}(\varepsilon)$ the density of perturbed states with angular momentum $J_{F}$. By making the simplification $P_{j_{N} J_{F}} \simeq P_{j_{N}}$, they will be evaluated from the simplest approximation

$$
P_{j_{N}}(\varepsilon)=\delta\left(\varepsilon-\Delta_{j_{N}}\right),
$$

for the ${ }_{\Lambda}^{5} \mathrm{He}$ states, whereas for ${ }_{\Lambda}^{12} \mathrm{C}$ they will be calculated from the Breit-Wigner distributions,

$$
P_{j_{N}}(\varepsilon)=\frac{2 \gamma_{j_{N}}}{\pi} \frac{1}{\gamma_{j_{N}}^{2}+4\left(\varepsilon-\Delta_{j_{N}}\right)^{2}},
$$

where $\gamma_{j_{N}}$ are the widths of the resonance centroid at energies $\Delta_{j_{N}}[29]$.

Finally, before to exhibit our numerical results, we would like to remark that the method developed here to include FSI can be applied independently of the exchange potential $V$ adopted for describing the NMHD. Particularly, we have employed the one-meson-exchange model from Refs. [20-22] which uses a standard strangeness-changing weak $\Lambda N \rightarrow N N$ transition potential comprising the exchange of the complete pseudoscalar and vector meson octets $\left(\pi, \eta, K, \rho, \omega, K^{*}\right)$.
The contribution of the meson $M$ to this potential depends on the radial functions [20]

$$
\begin{aligned}
f_{M}^{S}(r) & =\frac{1}{3}\left[m_{M}^{2} \frac{e^{-m_{M} r}}{4 \pi r}-\delta(\mathbf{r})\right] \\
f_{M}^{T}(r) & =\frac{m_{M}^{2}}{3}\left[1+\frac{3}{m_{M} r}+\frac{3}{\left(m_{M} r\right)^{2}}\right] \frac{e^{-m_{M} r}}{4 \pi r}, \\
f_{M}^{\prime}(r) & =-m_{M}\left(1+\frac{1}{m_{M} r}\right) \frac{e^{-m_{M} r}}{4 \pi r}
\end{aligned}
$$

with $f_{M}^{S}(r)$ and $f_{M}^{T}(r)$ describing the parity conserving scalar and tensorial parts, respectively, and $f_{M}^{\prime}(r)$ the parity violating one. Here it is also important to stress that we have included in our calculation the final state interactions between the two outgoing $n N$ particles, which have been extensively discussed in the literature and are known to be very important [13]. Their effect have been included phenomenologically by modifying the two emitted nucleons plane waves as in our mentioned works. In this way, final short range correlations are simulated at a simple Jastrow-like level multiplying the exchange potential by the correlation function

$$
g_{N N}(r)=1-j_{0}\left(q_{c} r\right),
$$

with $q_{c}=3.93 \mathrm{fm}^{-1}$. The finite nucleon size effects at the interaction vertices are gauged by the monopole form factor 
$\left(\Lambda_{M}^{2}-\mu_{M}^{2}\right) /\left(\Lambda_{M}^{2}+q^{2}\right)$, being $\Lambda_{M}$ the cutoff for the meson $M$ [20]. This corresponds in coordinate space to replacing each of the shape functions (13) as follows:

$$
\begin{aligned}
& f_{M}^{S}(r) \rightarrow \bar{f}_{M}^{S}(r)=f_{M}^{S}(r)-f_{\Lambda_{M}}^{S}(r)-\frac{1}{6}\left(\Lambda_{M}^{2}-m_{M}^{2}\right)\left(\Lambda_{M} r-2\right) \frac{e^{-\Lambda_{M} r}}{4 \pi r}, \\
& f_{M}^{T}(r) \rightarrow \bar{f}_{M}^{T}(r)=f_{M}^{T}(r)-f_{\Lambda_{M}}^{T}(r)-\frac{1}{6}\left(\Lambda_{M}^{2}-m_{M}^{2}\right)\left(\Lambda_{M} r+1\right) \frac{e^{-\Lambda_{M} r}}{4 \pi r}, \\
& f_{M}^{\prime}(r) \rightarrow \bar{f}_{M}^{\prime}(r)=f_{M}^{\prime}(r)-f_{\Lambda_{M}}^{\prime}(r)+\frac{r\left(\Lambda_{M}^{2}-m_{M}^{2}\right)}{2} \frac{e^{-\Lambda_{M} r}}{4 \pi r},
\end{aligned}
$$

where $f_{\Lambda_{M}}(r)$ has the same structure as $f_{M}(r)$ but with $m_{M} \rightarrow$ $\Lambda_{M}$. Additionally, we have taken into account corrections due to kinematical effects related to the $\Lambda$-nucleon mass difference and the first-order nonlocal terms [21].
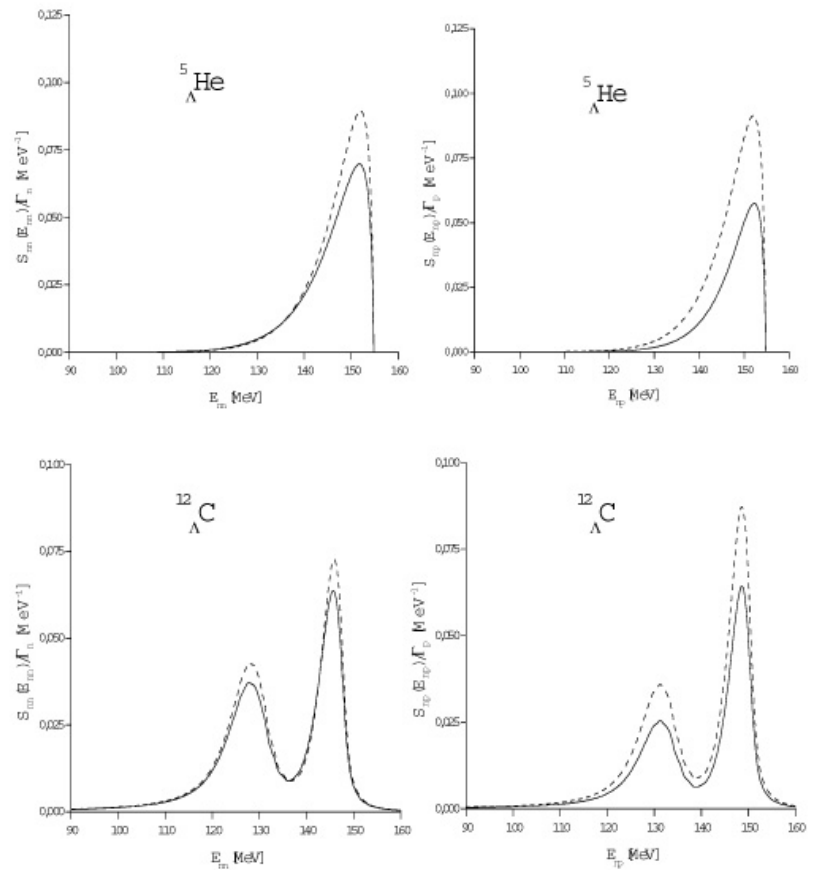

FIG. 1: Normalized energy sum spectra for ${ }_{\Lambda}^{5} \mathrm{He}$ and ${ }_{\Lambda}^{12} \mathrm{C}$. Solid and dashed lines correspond to calculations with and without FSI, respectively.

\section{RESULTS AND CONCLUDING REMARKS}

In the previous section we have developed a very simple and easily manageable procedure to analyze the effect of FSI on the kinetic energy sum spectra $S_{n N}(E)$. This method will be applied to evaluate the coincidence $n N$ spectra for ${ }_{\Lambda}^{5} \mathrm{He}$ and ${ }_{\Lambda}^{12} \mathrm{C}$ decays. With the aim of comparison, we have performed two evaluations of the spectra for each hypernucleus: one neglecting and the other including the effect of final interactions. When one neglects their influence at all we take
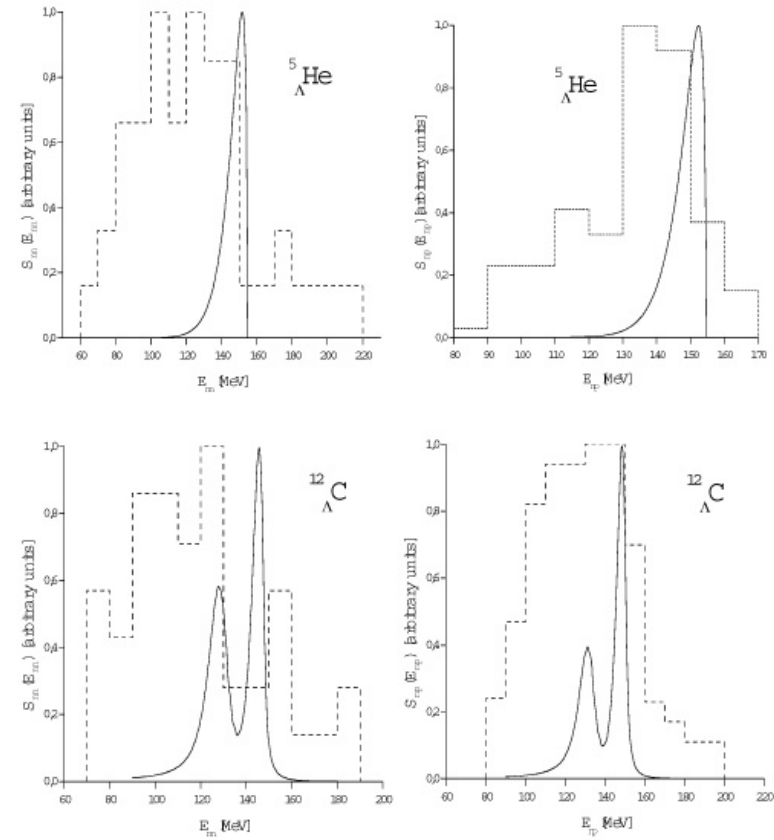

FIG. 2: Comparison between theoretical results including FSI (solid line) and experimental data (dashed lines) for the energy sum spectra of ${ }_{\Lambda}^{5} \mathrm{He}$ and ${ }_{\Lambda}^{12} \mathrm{C}$ decays.

$\tilde{V}_{0}=\tilde{V}_{1}=0$ in Eqs. (3) and (4). Otherwise, the calculation including FSI effect has been realized adopting the values carefully discussed in Ref. [19] for the potentials: $\tilde{V}_{0}=15$ $\mathrm{MeV}\left(\tilde{V}_{0}=20 \mathrm{MeV}\right)$ and $\frac{\tilde{V}_{1}}{2 A}=13.75 \mathrm{MeV}\left(\frac{\tilde{V}_{1}}{2 A}=5 \mathrm{MeV}\right)$ for ${ }_{\Lambda}^{5} \mathrm{He}\left({ }_{\Lambda}^{12} \mathrm{C}\right)$. We present in Fig. 1 our results for the normalized $S_{n N}(E) / \Gamma_{N}$ coincidence spectra of ${ }_{\Lambda}^{5} \mathrm{He}$ and ${ }_{\Lambda}^{12} \mathrm{C}$ hypernuclei. They show that the part of FSI we have considered until now in spite of giving a sizable contribution to the spectra do not have a decisive effect on it, in the sense that they do not modify qualitative its form. These FSI, included through the renormalization of the momenta $\mathbf{p} \rightarrow \alpha_{N} \mathbf{p}$ and $\mathbf{P} \rightarrow \alpha_{N} \mathbf{P}$ (see Eq. (2)) correspond to: i) admixtures between the states $\mid n n ; \mathrm{n}^{-1}>$ and $\mid n p ; \mathrm{p}^{-1}>$ (through the term proportional to $\tilde{V}_{1}$ in the optical potential) with $n n$ and $n p$ representing the 


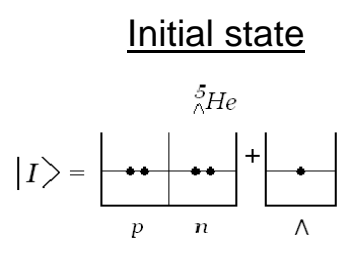

Final state

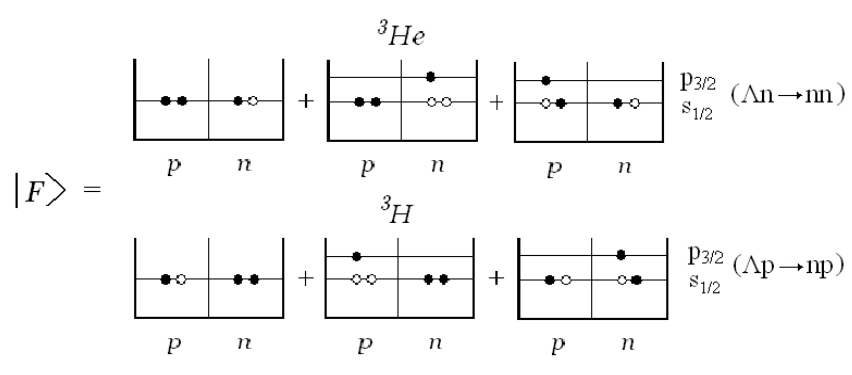

FIG. 3: Initial and final states for ${ }_{\Lambda}^{5}$ He nonmesonic decay.

pair of outgoing nucleons and $\mathrm{n}^{-1}\left(\mathrm{p}^{-1}\right)$ the neutron (proton) hole in the residual core; ii) mixtures of the states $\left|n n ; \mathrm{n}^{-1}\right\rangle$ and $\left|n p ; \mathrm{p}^{-1}\right\rangle$ with themselves (through the term proportional to $\tilde{V}_{0}$ ). With the purpose of helping to understand the physic involved in NMHD, we show in Fig. 2 our results for the kinetic sum spectra including FSI together with the experimental data taken from Refs. [8, 9], adequately normalized to facilitate the comparison. Our theoretical $n n$ and $n p$ spectra exhibit a series of peaks, one for each occupied shell model state. This agrees qualitatively well with the $n p$ data of ${ }_{\Lambda}^{5} \mathrm{He}$ where a bump at $\sim 145 \mathrm{MeV}$ is shown but the concordance for $n n$-coincidences measurements is no so good because a low energy bump is exhibited by the data. This in- dicate that our description of the mechanism involved in the NMHD is incomplete. In the case of ${ }_{\Lambda}^{12} \mathrm{C}$ spectra (or heavier hypernuclei) because of the resolution of the detector system, not considered here, is reasonable that only a wide bump at about $130 \mathrm{MeV}$ would appear in the experimental spectra. This problem could be eliminated after the forthcoming data of the experiments at J-PARC. Meanwhile, the ${ }_{\Lambda}^{5} \mathrm{He}$ data offer a good opportunity to improve our description of NMHD. It is interesting to mention here that similar comments can be drawn about more evolved evaluations of coincidence spectra including FSI effects at one-hole excitations level [30].

Therefore, in order to analyze future improvements to achieve a complete description of FSI felt by the outgoing nucleons, we will consider a simple 'toy model' including the possibility of more energetic configurations in the final state, i.e., configurations containing additional particle-hole $(p h)$ excitations generated by FSI of the emitted nucleons and absent in the eikonal picture. We show in Fig. 3 the initial and final states contributing to the NMHD of ${ }_{\Lambda}^{5} \mathrm{He}$, where the core is $\left|J_{C} M_{C}\right\rangle=|0\rangle \equiv\left|{ }^{4} \mathrm{He}\right\rangle$. Considering the $2 h 1 p, 3 h 2 p, \cdots, \kappa h(\kappa-1) p, \cdots$ configurations as perturbations over the $1 h$ one, originated by nuclear interaction between the emerging $n N$ nucleons and those in residual core, the time independent perturbation theory gives the available final states as

$$
\left|\overline{n N ; \kappa}>=\sum_{\kappa^{\prime}=1,2, \cdots N^{\prime}=n, p} C_{\kappa \kappa^{\prime}}\left(N N^{\prime}\right)\right| n N^{\prime} ; \kappa^{\prime}>, \kappa=1,2, \cdots,
$$

where $\mid n N^{\prime} ; \kappa^{\prime}>$ represents the unperturbed state with a $\kappa^{\prime} h\left(\kappa^{\prime}-1\right) p$ excitation and $\mid \overline{n N ; \kappa}>$ the corresponding perturbed one. To simplify we will restrict to additional $2 h 1 p$ excitations considering only the following configurations: $\left|n n ; \mathrm{n}^{-1}, \mathrm{nn}^{-1}>,\right| n n ; \mathrm{n}^{-1}, \mathrm{pp}^{-1}>, \mid n p ; \mathrm{p}^{-1}, \mathrm{nn}^{-1}>$ and $\mid n p ; \mathrm{p}^{-1}, \mathrm{pp}^{-1}>1$. In this case the unperturbed final states will be

$$
\begin{aligned}
|n N ; 1\rangle & \equiv b_{J_{F} M_{F}, N}^{\dagger}|0 ; n N\rangle \\
|n N ; 2\rangle & \equiv\left[b_{p_{\frac{3}{2}}, N^{\prime}}\left(b_{s_{\frac{1}{2}}, N^{\prime}}^{\dagger} b_{s_{\frac{1}{2}}, N}^{\dagger}\right)_{J^{\prime}=0,1}\right]_{J_{F} M_{F}}|0 ; n N\rangle, \quad \text { for } \quad N^{\prime}=n, p,
\end{aligned}
$$

where $b_{j, N}^{\dagger}\left(b_{j, N}\right)$ creates (destroys) an $N$-hole in the single particle state $\mathrm{j}_{N}$. The final state $\mid n N ; J_{F}>$ will be one of the perturbed levels $|\overline{n N ; 1}\rangle$ or $|\overline{n N ; 2}\rangle$ given in Eq. (16). The coefficients containing the information about nuclear interaction, $V_{\text {strong }}$, are given by $C_{\mathrm{\kappa \kappa}^{\prime}}\left(N N^{\prime}\right)=\frac{<n N^{\prime} ; \mathrm{K}^{\prime}\left|V_{\text {strong }}\right| n N ; \kappa>}{E_{\mathrm{K}}-E_{\mathrm{K}^{\prime}}}$ for $\kappa \neq \kappa^{\prime}$ and $C_{\kappa \kappa}\left(N N^{\prime}\right)$ is fixed by the normalization condition $\sum_{\mathcal{K}^{\prime} N^{\prime}}\left|C_{\mathrm{K \kappa}^{\prime}}\left(N N^{\prime}\right)\right|^{2}=1$. Introducing these final states in the nuclear moment $\left\langle J_{C}\left\|a_{\mathrm{j}_{N} m_{t_{N}}^{\dagger}}^{\dagger}\right\| J_{F}\right\rangle$ from Eq. (7) it is easy to see that only the terms proportional to the state $|n N ; 1\rangle$ will give a non-null contribution. As a consequence, the coincidence spectra will receive now contributions coming from the configuration $|\overline{n N ; 1}\rangle$ (proportional to $C_{11}$ ) but also from $|\overline{n N ; 2}\rangle$ (proportional to $C_{21}$ ). Thus, we can schematically write

$$
S_{n N}(E)=\left|C_{11}(N N)\right|^{2} S_{n N}^{\mathrm{lh}}(E)+\left|C_{21}(N N)\right|^{2} S_{n N}^{\mathrm{hhlp}}(E),
$$

where $S_{n N}^{\mathrm{th}}(E)$ is given by Eqs. (10)-(12) with $\Delta_{j_{N}}=\varepsilon_{j_{\Lambda}}+$ $\varepsilon_{j_{N}}+\Delta$ and $S_{n N}^{2 \mathrm{hlp}}(E)$ has exactly the same form but with $\Delta_{j_{N}}=\varepsilon_{j_{\Lambda}}+\varepsilon_{j_{N}}+\Delta+\Delta_{t r}$ being $\Delta_{t r}=\varepsilon_{s_{\frac{1}{2}}}-\varepsilon_{p_{\frac{3}{2}}}$ the energy invested in the $s_{\frac{1}{2}} \rightarrow p_{\frac{3}{2}}$ transition. Because $\varepsilon_{p_{\frac{3}{2}}}^{2}>\varepsilon_{s_{\frac{1}{2}}}$ the new $2 h 1 p$ contribution tends to remove nucleons from the high energy part of the spectrum while filling the low energy region. The intensity of the new peak at lower energies will depend of the value of the coefficients $C_{\kappa 1}$. Its evaluation is out of the scope of the present work but our reasoning indicates that a future detailed evaluation could shed some 
light on the physics involved in the problem. In fact, additional nuclear excitations produced by FSI could help to explain the discrepancies between theoretical and experimental data for the coincidence spectra and also the differences observed between $n n$ and $n p$ spectra. Because we are adopting a very simplified toy model our conclusions are not definitive. Additionally, we are neglecting at all the contribution of two-nucleon-induced decay, which could also produce nucleons to lower energies, mainly because the available energy is shared between three final particles.

In summary, we have performed a simple analysis of the effect of final state interactions on the $n N$ spectra as a function of the kinetic energy sum of the outgoing nucleons, $S_{n N}\left(E=E_{n}+E_{N}\right)$. We have studied the effect of FSI adopting both: i) the formalism developed in Ref. [16] for the calculation of the $n N$-coincidence spectra, which offers an adequate scheme to appraise the effect of additional contributions not included in the plane independent particle shell model; and ii) a simple method based on the eikonal approximation recently developed in Ref. [19] to incorporate the effect of the interactions felt by the outgoing nucleon in its travel through the residual core. We show that these FSI, which mainly produce a renormalization of the nucleon's momenta originated from the rescattering processes, give an important contribution but do not produce the desired effect on the spectra, in the sense that they do not generate the bump observed in the experimental $n n$ spectrum of ${ }_{\Lambda}^{5} \mathrm{He}$ at $\sim 100$ $\mathrm{MeV}$. Our results indicate that this low energy peak could be originated from more energetic configurations which should be added to the final state interactions considered until now in our formalism. A detailed evaluation of the effect of such configurations and also from two-nucleon-induced decay within the framework of finite nucleus formalism is required in the future.

\section{Acknowledgments}

C.B. and A.M. are fellows of the CONICET, CCT La Plata (Argentina) and thank to the Agencia Nacional de Promoción Científica y Tecnológica for supporting this work through PICT-2007-00861. S.B.D. thanks to Brazilian agency CNPq for partial support.
[1] J.H. Kim et al., Phys. Rev. C68, 065201 (2003).

[2] S. Okada et al., Phys. Lett B597, 249 (2004).

[3] M. Agnello et al., Nucl. Phys. A804, 151 (2008).

[4] J.D. Parker et al., Phys. Rev. C76 035501 (2007).

[5] M. Agnello et al., arXiv: nucl-th/09104939, (2009).

[6] S. Okada et al., Nucl. Phys. A752, 169c (2005).

[7] H. Outa et al., Nucl. Phys. A754, 157c (2005).

[8] B.H. Kang et al., Phys. Rev. Lett. 96, 062301 (2006).

[9] M.J. Kim et al., Phys. Lett. B641, 28 (2006) 28.

[10] H. Bhang et al., Eur. Phys. J. A33, 259 (2007).

[11] M.J. Kim, Private Communication (2009).

[12] A. Ramos, M.J. Vicente-Vacas and E. Oset, Phys. Rev. C55, 735 (1997); A. Ramos, M.J. Vicente-Vacas and E. Oset, Phys. Rev. C66, 039903 (E) (2002).

[13] A. Parreño and A. Ramos, Phys. Rev. C65, 015204 (2002).

[14] W. M. Alberico and G. Garbarino, Phys. Rep. 369, 1 (2002).

[15] G. Garbarino, A. Parreño and A. Ramos, Phys. Rev. C69, 054603 (2004).

[16] C. Barbero, A.P. Galeao, M.S. Hussein and F. Krmpotić, Phys. Rev. C78, 044312 (2008).

[17] E. Bauer, A.P. Galeao, M.S. Hussein, F. Krmpotić and J.D. Parker, Phys. Lett. B674, 109 (2009).

[18] F. Conti, A. Meucci, C. Giusti and F.D. Pacati, arXiv: nuclth/09123630, (2009).
[19] C. Barbero, A. Mariano and S.B. Duarte, arXiv: nuclth/09101127, (2009).

[20] C. Barbero, D. Horvat, F. Krmpotić, T.T.S. Kuo , Z. Narančić and D. Tadić Phys. Rev. C66, 055209 (2002).

[21] C. Barbero, C. de Conti, A.P. Galeao and F. Krmpotić, Nucl. Phys. A726, 267 (2003).

[22] C. Barbero, A.P. Galeao and F. Krmpotić, Phys. Rev. C72, 035210 (2005).

[23] G.P. McCauley and G.E. Brown, Proc. Phys. Soc. London 71, 873 (1958)

[24] C.J. Joachain, Quantum Collision Theory (Elsevier, Amsterdam, 1975).

[25] R.D. Amado, J. Piekarewicz, D.A. Sparrow and J.A. McNeil, Phys. Rev. C28, 1663 (1983).

[26] D. Debruyne, J. Ryckebusch, W. Van Nespen and S. Janssen, Phys. Rev. C62, 024611 (2000).

[27] L.D. Landau and E.M. Lifshiz, Mecánica Cuántica no relativista (Reverte S.A., 1972) p. 181.

[28] B. Van Overmeire, W. Cosyn, P. Lava and J. Ryckebusch, Phys. Rev. C73, 064603 (2006) and references therein.

[29] C. Mahaux, P.E. Bortignon, R.A. Boglia and C.H. Dasso, Phys. Rep. 120, 1 (1985).

[30] E. Bauer, Nucl. Phys. A796, 11 (2007). 